www.jmscr.igmpublication.org

Impact Factor 5.84

Index Copernicus Value: 83.27

ISSN (e)-2347-176x ISSN (p) 2455-0450

crossref DOI: https://dx.doi.org/10.18535/jmscr/v5i3.143

Journal Of Medical Science And Clinical Research

\title{
Comparative Analysis of Computed Radiography and Direct Digital Radiography Imaging and Their Artifacts
}

\author{
Authors \\ Ritu Kela (PhD) ${ }^{1}$, Nida Khan (M. Tech $)^{2}$, Anand Priyadarshi (B.Sc) ${ }^{3}$ \\ ${ }^{1}$ Department of Bio-Chemistry, College of Applied Education and Health Sciences, Meerut, India \\ Email:dean@caehs.edu.in \\ ${ }^{2}$ Department of Bio-Technology, College of Applied Education and Health Sciences, Meerut, India \\ Email: nidak.881@ gmail.com \\ ${ }^{3}$ Department of Radiology, College of Applied Education and Health Sciences, Meerut, India \\ Email:anand89uk@gmail.com
}

\begin{abstract}
Computed radiography $(C R)$ systems are used to perform radiographic examinations. CR systems possess various advantages over conventional radiography and are recognized globally. CR systems are facing strong competition with the introduction of more advanced and completely digital, direct digital radiography $(D D R)$ systems. Both the modalities have some or the other advantages over each other. Still, direct digital radiography $(D D R)$ systems is increasingly gaining the popularity and probably will take-over the CR systems in near-future. The common disadvantage with these modalities is an artifact Artifacts may occur on both the CR and the DR images and can degrade or mimic the clinical features. Several factors affect the image quality and contribute to the formation of artifacts. To optimize the image quality, it is important to understand the cause of artifacts and how they can be prevented. Despite the latest advancements in digital imaging, quality assurance and proper exposure technique is still a crucial challenge in radiography. The aim of this paper is to discuss and review the computed radiography and its artifacts to comprehend and understand the same for a better qualitative radiographic results.

Keywords- Artifact, Exposure, Computed-radiography, Medico-legal.
\end{abstract}

\section{Introduction}

Computed Radiography (CR) was first introduced by Fuji photo film. Fuji patented and commercialized the first CR system using Kodak technology in $1983^{[1]}$. The CR systems are accepted and recognized globally since three decades and are widely available all over the world. They are reliable and flexible in variety of clinical procedures. CR systems were strongly challenged by DDR systems when Selenium- based and Gadolinium-based (scintillator) flat panel detectors (FPD) were introduced in 1995 and 1997 respectively and have been used for the portable flat panel detectors since $2001^{\text {[2] }}$.

Imaging facilities are shifting to DR equipment due to its inherent work flow efficiencies. Many facilities are encountering artifacts similar to CR in addition to new ones, which are a direct result of the way the DR images are generated. Apart from this, classical technical errors (such as 
malpositioning, patient motion, incorrect patient identification, incorrect examination and double exposure etc still occur in the usual frequency. An understanding of the causes of both new and old problems is necessary so much to avoid these inaccuracies and overcome the problems that could jeopardize patient safety. On the clinical side, radiographer should be aware of about the unique conditions that apply to DDR operation [3,4]. X-rays were discovered by a German physicist Wilhelm Conard Roentgen on November 08, 1895 and introduced in medical diagnosis when a British physician John Francis HallEdwards first used the X-rays under clinical conditions on January $11,1896^{[5]}$. Since then medical imaging technology achieved the greatest height of advancement such as, 128 slice CT, 3T MRI, PET-CT and PET-MRI etc,. All these innovative modalities are mainly beneficial in diagnosing various cancers, neurological and cardiac deformities and other advanced diseases. Whereas the computed radiography generally associated with routine medical investigations in variety of diseases and, frequently used in chest and skeletal system radiography. Physiotherapy and Orthopedics or, general medicine to routine surgical procedures, all firmly rely on CR, as far as imaging is concerned. Latest technical updates and applications such as, increased detective quantum efficiency (DQE), higher spatial resolution, automated CR system, newer phosphor for PSP plates and, software enhancements are noted to increase the relevance of CR systems and are turning it more advanced and affordable than ever.

\section{CR image acquisition and processing}

Computed radiography, instead of conventional radiographic films-screens, uses a imaging plate (IP) made up of a photostimulable phosphor (PSP) material. These plates are coated with europiumactivated barium flurohalide (BAFx. $\mathrm{Eu}^{2+}$ ). The halide may be bromide or iodide or combination of both. The imaging plate is enclosed in a cassette and exposed in a manner similar to screen film cassette using with standard radiographic equipments as in conventional radiography. The PSP in plate absorbs x-rays that have passed through the patient and, as a result, the phosphor electrons become 'excited' and are raised to a higher energy level; these excited electrons stay trapped in a semi-stable, higher-energy state and represent a latent image on the phosphor plate in the form of stored energy. This latent image instead of taken in darkroom for developing and processing in chemical solutions, brought directly for scanning and processed by a laser-enabled CR reader that acquires image information. The stored energy is released by adding energy to the trapped electrons. This is done by stimulation with a laser beam during processing. The trapped electrons become free and return to their equilibrium state (lower energy level). During this process electrons release energy in the form of light. This is known as photo stimulable luminescence (PSL). The emitted light travels through a fiber-optic light guide to photomultiplier tube (TMT) where light is converted into electronic signal. This signal from PMT is digitized and stored and displayed on a computer system and available for clinical diagnosis. The digital image can be easily printed or transmitted to picture archiving and communication system (PACS). The phosphor plate is then erased with a bright light to remove any remaining trapped electrons and the imaging plate (IP) is then ready for the next examination ${ }^{[6,7]}$.

\section{Comparison with DR}

DDR systems achieves better images at low radiation levels with less dependency on the skills of the radiographer. However, DR is more expensive and its images are marginally better whereas $\mathrm{CR}$ is cost effective and its results are already good enough for clinical diagnosis. In India the majority of the hospitals do not have portable DDR systems, so, mobile CR systems are the instant choice for emergency and bedside radiography. Moreover, as DDR detectors are not free to place in all positions, some radiographic views are not possible. Whereas CR cassettes can 
be placed in any position specially in ICU and NICU radiography where high degree of care and dedication is required to serve the critical patients during radiographic examinations without compromising patient care and image quality with minimum exposure risks. Though DDR possess some advantages (such as; various auto selections, automatic tube detector positioning, increased work flow and quality control of images for instant diagnosis) over CR but it has not entirely free from the artifacts. Different DR system model and usage have the prospect for a different manifestation or degree of artifact. Even if vendors use the same physical detector hardware, differences in calibration or image processing may yield different results or risk for artifacts. FPD, which is the most promising feature of DR system generally allow a shorter time interval between exposures compared with cassette-based CR system. Incidentally, most systems can acquire images at a rate faster than their detectors can actually accommodate. The rapid acquisition of images can result in latent signal from one exposure lingering into the read-out of subsequent exposures, producing what appears to be an incomplete erasure of the previous image, known as imaging lag. These image lags can be occur as image artifacts in clinical practice ${ }^{[3]}$. The terms "lag" and "ghosting" are often used interchangeably, can also occur with CR system. The image quality of CR on large body parts, such as chest and abdomen, is better than that of DR, whereas DR is preferred for small body parts and extremities ${ }^{[8]}$. Unlike DDR, a multiple cassette size option is also available in CR. Even though digital radiography is gaining in popularity $\mathrm{CR}$ will continue to play a significant role in an emergency setup. After performing cost analysis and capacity utilization of general radiography, CR still scores over digital radiography ${ }^{[9]}$.

\section{Artifacts or Artefacts}

It's bit confusing but both have identical meaning. The main difference is that in USA it is called as artifact whereas British spelled it as Artefact.
Artifact is an artificial structure on radiographic image that is not occurring naturally but, produced by the technique used or due to any external source. They are undesirable and not present in the original object. Some artifacts may be confused with pathology during clinical evaluation, while others can seriously degrade the diagnostic quality. Errors by radiographers increase the risk of false diagnosis and may harm the patients and eventually lead to medico legal issues. A survey analysis in 1503 German radiologist showed that radiography (20.2\%), angiography (18.4\%), and, mammography (16.4\%) were the most frequent imaging techniques which underwent law proceedings that led to civil $(30 \%)$ or criminal $(5.5 \%)$ convictions ${ }^{[10]}$.

\section{Computed Radiography Artifacts}

Various factors affect the quality of image and CR artifacts can be produced at any stage during image acquisition and image processing.

\section{Image Acquisition Artifacts}

These artifacts are due to operating errors by radiographers with improper radiographic techniques, such as incorrect collimation, double exposure, exposure technique artifacts, grid artifacts and light bulb effect.

\section{Operating Errors}

These errors are common when CR system is new to radiographers or there are some faults in system installation and their maintenance. Rough handling of cassette should be avoided and used with utmost care and stored properly. The cassette must be protected from heat, low humidity, ionizing and scattered radiations to avoid any damage to sensitive IP, which is enclosed in the cassette. The cassette orientation for any receptor is an important detail. Since various cassettes are designed and constructed differently, each version leaves its own pattern of artifacts, if the cassette exposed upside down ${ }^{[11]}$. To avoid upside down cassette artifacts, knowledge of cassette orientation is essential. Long working hours are 
also contribute to operating errors and can seriously affect the professional efficiency of the radiographer. Those who are working in 12 hours shifts, sometimes have to follow an additional day or night shift. The fatigue due to continuous working may lead to serious radiographic errors and could result in a serious injury to the patient and the radiographer. It also increases the risk of occupational radiation exposure. It's the responsibility of the employer to fill the adequate radiographic positions with suitably-trained radiographers to manage such kind of operative errors.Although, most of the operating errors artifacts involved a minor degree of radiographic negligence and can definitely be resolved, if a sincere and professional approach followed strictly.

\section{Incorrect Collimation}

Incorrect collimation can causes inappropriate image processing, scattering and failure to detect radiation field (exposure recognition failure) resulting in an incomplete and irrelevant image with poor quality. For instance, when lumbosacral (LS) spine isradiographed, without collimation, it appears like an image of the Kidney-UreterBladder (KUB) technique. Causes include non parallel collimation, multiple fields, poor centering, implants and violation of basic rules. Incorrect collimation can be prevented with proper collimation and appropriate positioning ${ }^{[12]}$.

\section{Double exposure}

Double exposure is a classic operator error that constitutes approximately $2 \%$ of all rejected images. The image may appear superimposed. The consequence of double exposure can be either a single repeated examination, when an inanimate object is involved or two repeated examinations when two patients are involved ${ }^{[4]}$. Basic understanding of radiographic equipments is crucial to reduce such corrupt repetitions.

\section{Delayed Scanning}

A delay between acquisition and processing of image will lead to fading of the image. $25 \%$ of the stored signals will be lost in about 10 minutes to eight hours after exposure and, more slowly afterward ${ }^{[8,13]}$. The delay between exposure and processing should be as minimal aspossible to prevent such kind of artifacts.

\section{Exposure Techniques}

Exposure technical errors constitute following artifacts;

Overexposure- Due to high exposure factors image is overexposed and appears too dark with extra noise. Overexposed images with poor visibility may lose clinical features while some images can't be evaluated and lose their clinical value.

Underexposure- The exposure factors remains too low resulting as an underexposed, quantum mottle image with reduced signal-to-noise ratio with less clinical value. However, no imaging method is free of noise, all medical images contain some visual noise. The presence of noise gives an image a mottled, grainy textured or snowy appearance ${ }^{[14]}$. The exposure must be set to match the sensitivity of the receptors. Appropriate radiographic technique with proper factors (such as, $\mathrm{kVp}$, mAs, SID) must be used to reduce these artifacts. The exposure technique should always be based on ALARA ( "As low as reasonably Achievable.") principle.

\section{Grid artifacts}

Anti scatter grids are used in CR system for contrast enhancement that can produce specific artifacts in digital image. Currently stationary linear grids are most usable. Grid may causes Moire effect. In Moire pattern, grid lines run in the same direction as the laser in the CR reader. 33 lines per inch (LPI) grids have same frequency as CR laser scanner. This can cause Moire pattern artifact. To prevent this artifact, align grid lines 
perpendicular to scan oreintation whenever possible. It reduces the chances of artifacts caused by laser scanner. The implemented grid artifact detection and suppression method tested with different frequencies $(85,103,152$ LPI) and ratios $(6,8,10 \& 12)$ scanned orthogonally with pixel size of $0.171,0.115$ and $0.097 \mathrm{~mm}$. There was no failure on grid detection and processed images revealed effective grid artifacts suppression preserving acceptable image diagnostic quality as estimated by radiologists ${ }^{[6,15]}$.

\section{Light bulb effect}

The lower, outer portion of a film occasionally appears darkened relative to the remainder of the image, referred to as light bulb effect. It is caused by back-scattered radiations entering the PSP imaging plate from the patient's bed. This artifact is seen most frequently when the exposure is increased for obese patients or when the x-ray beam hasn't been collimated to the region of interest. This artifact could be misconstrued as pneumothorax or pneumoperitoneum in a supine patient with chest radiography. This artifact can be prevented with reducing backscatter by lowering kilovoltage $(\mathrm{kV})$ with accurate collimation ${ }^{[16]}$.

\section{Image Processing Artifacts}

These artifacts produced during image processing. Such as, CR reader artifacts, IP artifacts and, software related artifacts.

\section{CR reader artifacts}

These artifacts typically display as lines across the entire image in contrast to dirt within the cassette or on PSP which are shorter or curved lines.

\section{Printer distortion}

It occurs when the image size and the printed size are not equal. Artifacts caused by the laser printer on the printed image will not display on digital image. The light guide collects light from the imaging plate during laser scanning. Dirt on the light guide can creates artifacts. To prevent CR reader artifacts, dust, dirt or phantom (non-erased) images should be corrected with cleaning and plate erasure. Laser power will diminish over the time to a point beyond correction, necessitating replacement of the laser subsystem ${ }^{[6,17]}$.

\section{Imaging plate (IP) artifacts}

IP artifacts are produced due to scratches, scuffs, cracks and contamination with dust and dirt and affect the image quality. An IP has a maximum life of about 10000 exposures and should be replaced before its deterioration. The damaged IPs will only be contribute to artifacts \& eventually the repetition of exposure. Hence, replacement is the only solution. Excessive cleaning of the IP should be avoided even with approved cleaners to protect IP coating. A well organised quality control program for assessing the clinical quality of the imaging plate is essential ${ }^{[18,19]}$.

\section{Software related artifacts}

The software used to acquire, view and adjust the raw image may also cause some types of artifacts. Software malfunctions can lead to reconstruction algorithm's inability to represent the part or apply incorrect great scale values creating small, faint lines. ${ }^{[18]}$. Excessive edge enhancement may influence interpretation and can simulate pathology such as osteolytic lesions or osteomyelitis. Other software causes includes inappropriate display protocol selection, which often leads to incorrect display of the image. If image quality is not improved with customized contrast and sharpness or to confirm any questionable areas, the image should be repeated ${ }^{[20]}$. To prevent these artifacts, access to latest and timely updated image-processing software is essential to develop and maintain appropriate processing parameters.

\section{Patient Based Artifacts}

The most common patient related artifact is patient's motion; other include metallic implants, such as dental fillings, prosthetic devices and various foreign objects. 


\section{Motion artifacts}

Patient motion has devastating effect on image quality. Incomplete contact or distance between cassette and moving patient during radiography exam turns the image blurred with poorly visible features. Blurring can also be occurs from cassette or x-ray tube movements. Sources of motion artifacts may be voluntary (head motion, limbs motion, wrong posture), or involuntary (respiratory motion, cardiac pulsations, tremor and shivering). Motion artifacts can be prevented with the use of positioning aids and, appropriate use of radiography equipments with minimum time of exposure. Respiratory motion can be minimized if patients are able to hold their breath for few seconds during the exposure.

\section{Foreign object artifacts}

If any kind of foreign object outside or inside the body such as jewellery or metal denture retained with patient, it will create artifacts (double or ghost images) and must be removed before radiographs are taken ${ }^{[21]}$. Placement of gonadal shield during radiography is not beneficial and adversely affects the image quality. Therefore should not be used to avoid the artifacts. Patients with non-removable implants such as, dental fillings, prosthetic devices, surgical clips/sutures and cardiac conduction devices (CCDs) must be radiographed with appropriate imaging techniques.

\section{Surreptitious Image deletion}

Surreptitious deletion of non-diagnostic images is a negative practice and should be avoided by the radiographers. The image may contains some information and can be compared with re-exposed image. Further study and analysis could be helpful to understand artifacts and image repetition.

\section{Conclusion}

CR and DR systems, both have their own specific advantages and disadvantages. DR is completely digital and time saving, whereas, CR has a major advantage in ward radiography with its flexibility and reusable phosphor plates. Both systems possess their own kind sets of artifacts, some of them can be reduced with latest hardware and software updates. However prevention of operator error artifacts can only be achieved when certain fundamentals of medical imaging are followed during image acquisition. Appropriate radiographic techniques will reduce these errors. For a radiographer, it is not possible to know all the physics and technology behind these systems but, some basic knowledge of technology and pathology is necessary to review the image before sending it to clinical evaluation. To identify and reduce the errors and artifacts, appropriate training and education of radiographers is important and should be mandatory before operating any upgraded imaging modality and, CR or DR systems.

\section{Acknowledgements}

This review paper was strongly supported by Dr. S P Deshwal (Ph.D Zoology). We thank our colleagues from College Of Applied Education And Health Sciences who provided insight and expertise that greatly assisted in compiling this review. We thank Dr. S K Garg for assistance and sharing his pearls of wisdom with us during the course of this review paper.

\section{References}

1. Fujifilm Corporation, Fuji Computed Radiography (FCR).

2. Markus Korner, MD, Christof H. Weber, MD, Stefan Wirth, MD, Klaus-Furgen Pfeifer, MD, Maximilian F. Reiser, MD, Marcus Treitl, MD : Advances in Digital Radiography : Physical Principles and System Overview. RadioGraphics 2007; 27: 675-686 - published online 10.1148/rg. 273065075. Contentcode : PH.

3. Alisa Walz-Flannigan, Dayne Magnuson, Daniel Erickson, Beth Schueler : Artifacts in Digital Radiography - American Journal of Roentgenology 2012. 198 : 156-161. 
4. Willis CE : Artifacts and misadventures in digital radiography. Society of computer applications in radiology (SCAR), university course $305,20^{\text {th }}$ symposium Boston, MA, June 710, 2003.

5. Meggitt, Geoff (2008). Taming the rays : a history of radiation and protection.

6. Satish K. Bhargava, Textbook of Radiology for residents and technicians, $5^{\text {th }}$ edition. PP. 579-586, 641-648.

7. Clark's Positioning in Radiography. $12^{\text {th }}$ edition, PP 22-23.

8. Chandrakant Manmath Shetty, Ashita Barthur, AvinashKambadakone, Nilna Narayanan and RajagopalKv : Computed Radiography Image Artifacts Revisited American Journal of Roentgenology. 2011; 196 : W37-W47. 10.2214/AJR.10. 5563.

9. BI, Salkever D, Seigel EL Hooper FJ, Siddiqui KM, Musk A. - Multi-institutional analysis of computed radiography. Part II. Economic analysis. Radiology 2005; 236 : 420-426.

10. R. Tomczak, S. Schnabel, P. Ulrich, H.J. Brambs, N. Rilinger, and C. Nierhoff, "Frequency and causes of civil and criminal proceedings in radiology. Analysis of a survey."Radiologe, Vol.46, No.7, PP. 557-566, 2006.

11. LJ Cesar, RT(R)(QM), BA Schueler, $\mathrm{PhD}$, FE Zink, PhD, TR Daly, RT(R)(QM), JP Taubel, RT(R)(QM) and LL Jorgenson, RT(R). : Artefacts found in computed radiography. The British Journal of Radiology, 74 (2001), 195-202.

12. IAEA - Avoiding Artefacts in Computed Radiography, L06 - International Atomic Energy Agency.

13. Seibert JA, Bogucki T, Ciona T. Acceptance testing and quality control of photostimulable phosphor imaging systems. Report of task group 10.Version
3.1. College Park, MD : American Association of Physicists in Medicine, 1997.

14. Perry Sprawls, PhD. The Physical Principles of Medical Imaging : Sprawls educational foundation.

15. IgorBelykh. Grid Artifacts Suppression in Computed Radiographic Images. International Science Index, Biomedical and Biological Engineering Vol:8, No:8, 2014 waset.org/publication/9999139.

16. Steven L. Solomon, R. Gilbert Jost, Harvey S. Glazer, Stuart S. Sagel, Dixie, J. Anderson, and Paul L. Molina. American Journal of Roentgenology, 157 : 181-185, July 1991.

17. IAEA. Radiation Protection in Digital Radiology. : International Atomic Energy Agency.

18. J. Tyler Bouye : Computed Radiography and Artifacts. URS energy and construction Savannah river site - Mox Project Bldge 245-15F, Aiken, SC 29808.

19. Willis CE : What's new in quality assurance for computed radiography? Decisions in imaging economics. The Journal of Imaging Technology Management. October 2004.

20. LTH Tan and KL Ong : Artifacts in Computed Radiography. Hong Kong Journal of Emergency Medicine.Vol.7(1). Jan 2000.

21. Neill Serman. Radiographic Errors and Artifacts. August 2000. 